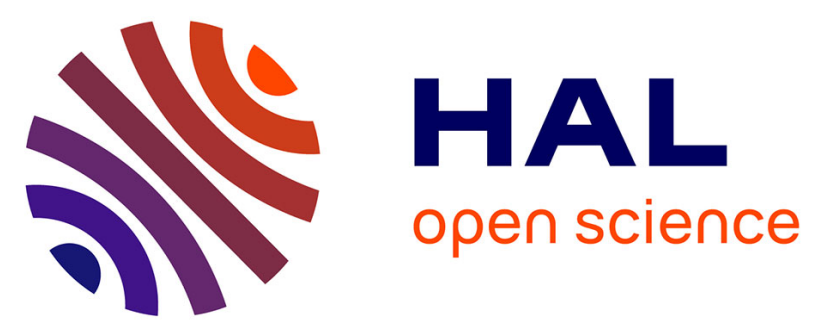

\title{
Bone-conducted sound in a dolphin's mandible: Experimental investigation of elastic waves mediating information on sound source position
}

Michael Reinwald, Quentin Grimal, Jacques Marchal, Stefan Catheline, Lapo Boschi

\section{To cite this version:}

Michael Reinwald, Quentin Grimal, Jacques Marchal, Stefan Catheline, Lapo Boschi. Bone-conducted sound in a dolphin's mandible: Experimental investigation of elastic waves mediating information on sound source position. Journal of the Acoustical Society of America, 2018, 144 (4), pp.2213-2224. 10.1121/1.5063356 . hal-01981636

\section{HAL Id: hal-01981636 \\ https://hal.sorbonne-universite.fr/hal-01981636}

Submitted on 15 Jan 2019

HAL is a multi-disciplinary open access archive for the deposit and dissemination of scientific research documents, whether they are published or not. The documents may come from teaching and research institutions in France or abroad, or from public or private research centers.
L'archive ouverte pluridisciplinaire HAL, est destinée au dépôt et à la diffusion de documents scientifiques de niveau recherche, publiés ou non, émanant des établissements d'enseignement et de recherche français ou étrangers, des laboratoires publics ou privés. 
Bone-conducted sound in a dolphin's mandible: Experimental investigation of elastic waves mediating information on sound source position

Michael Reinwald, ${ }^{1, a)}$ Quentin Grimal,, b Jacques Marchal, ${ }^{2, c}$ Stefan Catheline, ${ }^{3, \mathrm{~d}}$ and Lapo Boschi, e

${ }^{1}$ Sorbonne Université, CNRS, INSERM, Laboratoire d'Imagerie Biomédicale, LIB, F-75006, Paris, France.

${ }^{2}$ Sorbonne Université, CNRS, Institut Jean le Rond d'Alembert, F-78210, Saint-Cyr-l'École, France.

${ }^{3}$ LabTAU, INSERM, Centre Léon Bèrard, Université Lyon 1, Univ. Lyon, F-69003, Lyon, France.

${ }^{4}$ Sorbonne Université, CNRS-INSU, Institut des Sciences de la Terre Paris, ISTeP UMR 7193, F-75005 Paris, France.

(Dated: 29 September 2018) 
Mammals use binaural or monaural (spectral) cues to localize acoustic sources. While the sensitivity of terrestrial mammals to changes in source elevation is relatively poor, the accuracy achieved by the odontocete cetaceans' biosonar is high, independently of where the source is. Binaural/spectral cues are unlikely to account for this remarkable skill. We study bone-conducted sound in a dolphin's mandible, investigating its possible contribution to sound localization. Experiments are conducted in a water tank by deploying, on the horizontal and median planes of the skull, ultrasound sources that emit synthetic clicks between $45-55 \mathrm{kHz}$. Elastic waves propagating through the mandible are measured at the pan bones and used to localize source positions via either binaural cues or a correlation-based full-waveform algorithm. Exploiting the full waveforms and, most importantly, their reverberated coda, we can enhance the accuracy of source localization in the vertical plane, and achieve similar resolution of horizontal- vs. vertical-plane sources. Our results need to be substantiated by further experimental work, accounting for soft tissues and making sure that the data are correctly mediated to the internal ear. If confirmed, they would favor the idea that dolphin's echolocation skills rely on the capability to analyze the coda of biosonar echoes.

a) michael.reinwald@upmc.fr

b) quentin.grimal@upmc.fr

c) jacques.marchal@upmc.fr

d) stefan.catheline@inserm.fr

e)lapo.boschi@upmc.fr 
Reinwald et al., JASA

\section{INTRODUCTION}

The acoustic environment of marine mammals is very different from that of humans and other terrestrial mammals. Water is much denser than air, and sound travels five times faster through water than through air and is less strongly attenuated; the energy carried by acoustic waves is more efficiently transferred to bone tissue from water than from air; presumably because they would be disadvantageous from the point of view of locomotion in water, marine mammals have lost pinnae through evolution; their ear canals are typically filled with cellular debris and appear to play no functional role in hearing (Ketten, 1997).

Marine mammals use echolocation to navigate and hunt. For about two centuries (Hunter and Banks, 1787), they have been known to complete such tasks with remarkable accuracy and efficiency. The contribution of dolphin's anatomy to audition-related tasks was first evaluated by Kenneth Norris in a suite of groundbreaking studies (Norris, 1964, 1968a’b; Norris and Harvey, 1974). A dolphin's mandible is very thin, almost "translucent," at its posterior end ( $0.5 \mathrm{~mm}$ to $3.0 \mathrm{~mm}$ thickness, depending on the species), and is overlain by an oval fatty volume, which connects the posterior jaw bone, also named pan bone, with the tympano-periotic complex (TPC). Norris suggested that sound propagates through the thin pan bone, entering the fats which possibly act as a low-impedance wave guide that directs sound towards the inner ear. This is still the most widely accepted theory of the sound propagation pathway for hearing in cetaceans (Au, 2012; Au and Hastings, 2008; Brill et al., 2001; Mooney et al., 2012) and is supported by experimental (Brill et al., 1988; Norris and Harvey, 1974) and numerical (Aroyan, 2001) results. Norris' "jaw-bone theory" has been 
further developed in more recent studies: While high-frequency sounds could propagate through the jaw bone, low-frequency sounds (below $30 \mathrm{kHz}$ ) could propagate through bonefree fat channels just below the eyes and posterior to the lower jawbone (Brill et al., 2001; Ketten, 1994; Popov and Supin, 1990). Cranford et al. simulated sound propagation in the head of a Cuvier's beaked whale and introduced the idea of a "gular pathway" for sound reception (Cranford et al., 2008), with sound entering the head from below and between the mandibular walls rather than through the posterior mandible. Some studies propose that the teeth play an important role in sound reception; due to their periodic placement, they could act as an acoustic metamaterial, resonating and amplifying sound for specific frequencies (Dible et al., 2009; Graf et al., 2009). Others suggest that the morphology of the mental foramens found in the mandible helps sound enter the fatty tissues (Ryabov, 2010). Møhl et al. (Møhl et al., 1999) measured the auditory brainstem evoked potential for an Atlantic bottlenose dolphin by placing suction cups on various positions on the animal's head and emitting acoustic click stimuli via embedded piezoelectric transducer elements. Their results suggest that the area of maximum sensitivity is slightly forward of the pan bone area. This does not contradict Norris' jaw bone theory since the outer mandibular fat channel extends forward to the skin and towards the area where Møhl et al. found sensitivity to be highest.

It has been suggested by experiments (Blauert, 1997; Moore et al., 1995; Renaud and Popper, 1975) that dolphins locate sound sources via binaural cues known to be employed by terrestrial animals, i.e. interaural time differences (ITD), which describe the delay of a signal arriving at the two ears at two different times, and interaural level differences (ILD), which describe the difference in intensity between the signals perceived at the two ears. 
While ITD are relatively easy to reproduce theoretically, ILD effects are more complex, as they cannot be modeled by simply accounting for differences in source-receiver distance: they are importantly affected by sound shadowing due to the impedance mismatch between the subject's head and the surrounding propagation medium (Mooney et al., 2012), while a significant fraction of acoustic energy traveling from one ear to the other is diffracted by the head's surface, thus following a complex propagation path. In any case, binaural cues are only relevant as long as a sound originates from somewhere else than the median plane. Because median-plane sources are equidistant from both ears, no phase or amplitude (if the subject's anatomical features are symmetric with respect to the median plane, as they most often are) differences exist between the signals perceived at the two ears, i.e. zero ITD and ILD (e.g. Butler et al., 1990; Hartmann, 1999). ITD and ILD are naturally nonzero whenever the source is not on the median plane, so that the ears lay at different distances from the source.

The only (rare) cases of median-plane sources generating nonzero ITD/ILD are species characterized by asymmetrically positioned ears, e.g., the barn owl (Keller et al., 1998). Other species, including humans, are not very effective at differentiating sound source positions within the median plane (Butler and Belendiuk, 1969; Heffner and Heffner, 1992; Van Opstal, 2016), or, more generally, within a "cone of confusion" (e.g. Van Opstal, 2016). Their (limited) ability at this task must be explained in terms of non-binaural cues (e.g., acoustical clues not simply related to a difference between right and left signals). It has been suggested that sound-localizing animals learn to interpret certain acoustical cues associated with their anatomy in order to solve this ambiguity (Batteau, 1967; Blauert, 1969; Hart- 
mann, 1999; Macpherson and Sabin, 2013; Van Opstal, 2016). Anatomy can be thought of as a spectral filter (the head-related transfer function, or HRTF), which will change depending on source position: because the back of our head is different from our face, it interacts differently with an incoming wave field, which consequently sounds different to our ears. The HRTF associated with a human skull has been found to provide, in principle, sufficient information for a source to be localized with fairly high accuracy, independent of the location of the source, even when data from only one ear are used (Catheline et al., 2007); yet, psychoacoustics studies (Van Opstal, 2016) have shown that the performance of the, e.g., human ear-brain system at localizing median-plane sources is relatively poor: we are much more effective at discriminating sources within the horizontal plane. Other terrestrial species show the same limitations. It has also been found experimentally that humans are relatively poor at source localization tasks if only one ear is used; subjects with unilateral hearing loss apparently learn to function with one ear only, but their performance at sound localization has been found to remain significantly poorer than that of subjects with no hearing loss (Agterberg et al., 2011; Van Opstal, 2016). It is inferred that, while humans and other terrestrial species certainly use HRTF information in sound-localization tasks, they exploit only a subset of the information provided by the HRTF itself. The consensus is that the only monaural cues that they are actually capable of using are certain "notches" of the frequency spectrum perceived by the ears, or "spectral cues," whose amplitude, and location along the frequency axis, are controlled by the complex shape of the pinnae and depend on the position of the source (Van Opstal, 2016, Chapter 7). 
Cetaceans are also characterized by a salient HRTF (Aroyan, 2001; Au and Fay, 2012; Supin and Popov, 1993); how and to what extent they make use of it, is still unclear. Simple physical considerations suggest that anatomical features characterized by relatively strong density contrasts with respect to the surrounding medium (water) most significantly contribute to characterizing the HRTF, and thus to sound localization. Since the density of soft tissues found in marine mammal bodies is close to that of water (Norris and Harvey, 1974; Reysenbach de Haan, 1957), it is inferred that features such as the mandible, the cranium or small air sacs play the most important roles, similar to the external ears of terrestrial mammals (Aroyan et al., 1992; Song et al., 2017; Wei et al., 2016). One important difference in the sound localization performance of terrestrial mammals vs cetaceans is the latter's ability to localize sound sources within the median plane with a very high accuracy (Renaud and Popper, 1975). This can be quantified by the minimum audible angle (MAA), i.e., the minimum angular distance between two sources of sound, still allowing to discriminate them as two different sources. Signals emitted by two sources separated by an angle smaller than the MAA are perceived as coming from only one source. The MAA changes depending on the azimuth and elevation of the sources, and on the nature of the emitted signal. By studying the behavior of live dolphins when exposed to sound coming from different locations, their MAA in the median plane has been estimated around $0.7^{\circ}$ for broadband clicks. Similar values are observed for sources positioned on the horizontal plane (Au and Hastings, 2008; Nachtigall, 2016). In comparison, psychoacousticians estimate the MAA of human subjects at around $7^{\circ}$ in the vertical plane, as opposed to only $\sim 1^{\circ}$ in the horizontal one (Nachtigall, 2016), while other terrestrial mammals perform more poorly than humans 
(Heffner and Heffner, 2016, Figure 3). It can be inferred from these observations that, when echolocating, dolphins are capable of extracting from their HRTF more information than terrestrial mammals in sound localization tasks (Branstetter and Mercado III, 2006). The acoustic environment of cetaceans would indeed favor animals capable of localizing sound, whether it be emitted or reflected from prey or predators, regardless of their position in space. Dolphins' MAA grows to $2.3^{\circ}-3.5^{\circ}$ for narrow-band signals (Au and Hastings, 2008; Nachtigall, 2016), which do not mimic typical echolocation clicks.

This study addresses the question of how a dolphin's head inner anatomy may contribute to sound localization, and in particular to echo-localization, by means of a suite of physical acoustics experiments conducted on one skull specimen of short-beaked common dolphin (Delphinus Delphis). The HRTF of the short-beaked common dolphin has so far only been addressed in a limited number of studies. Most of our previous knowledge results from numerical models; Krysl and Cranford carried out vibroacoustic simulations on a CT scan of a full head of a common dolphin showing single-frequency HRTFs for $5.6 \mathrm{kHz}, 22.5 \mathrm{kHz}$ and $38 \mathrm{kHz}$ (Krysl and Cranford, 2016). In their work, single-frequency HRTFs were equivalent to amplitude or intensity variations with respect to the source position. They simulated sound pressure levels at two virtual positions on the surface of the TPCs (one dorsal, one ventral), caused by monochromatic plane waves traveling along a suite of different azimuths and elevation angles. Strong variations in modeled data were found between the dorsal and the ventral receiver positions. The spatial pattern of the HRTFs was also found to depend strongly on the source frequency used. The results were not conclusive as to what extent the calculated asymmetries in the receiving pressure pattern were due to the inexact placement 
of the receivers, versus asymmetries in the specimen's anatomy. Another, similarly minded study (Aroyan, 2001) showed that the mandible and its surrounding fats focus acoustic waves toward the TPC, therefore playing an important role in sound conduction through the head. Receptivity patterns at two virtual ear positions showed high asymmetry and complexity, and varied significantly depending on which parts of the head were simulated.

In our experimental study, we attempt to evaluate the specific contribution of bone conduction to sound localization. To this goal, we conduct experiments on a dolphin's skull specimen immersed in water, in the absence of soft tissues. It has been suggested (e.g., Aroyan, 2001; McCormick et al., 1970; Song et al., 2016; Wei et al., 2018) that bone conduction affects significantly the dolphin's HRTF. Bone conduction seems to be a driving force for hearing mechanisms in baleen whales (Cranford and Krysl, 2015). Despite the isolation of the skull from the TPC, Ketten also theorized some influence of bone conduction in dolphin hearing, i.e., on sound reception pathways (Ketten, 2000). Cranford et al. suggested that a complex wave propagation pattern including flexural waves along the mandible bone of a Cuvier's beaked whale might contribute to the received pressure at its two ears (Cranford et al., 2008). In summary, while it is likely that the mandible plays an important role in this context, the exact nature of its contribution to sound localization remains to be determined.

Using accelerometers glued to the pan bone, we measure elastic waves traveling through a mandible specimen immersed in water; we record the signal generated by different sound sources, positioned at many different locations within a large water tank; we measure the ITD and ILD (binaural cues) resulting from such recordings and estimate their potential performance as source-localization cues. Finally, we study in much detail how the wave- 
form of the recorded signal depends on source position, and use a correlation-based method (known in physical acoustics as "acoustic time reversal" (Fink et al., 2000)) to numerically reconstruct the location of sources via full-waveform data. In this endeavour, we take the standpoint of physicists, attempting to quantify the information carried by our data, independent of how these would be received and processed by the auditory system of a living organism. Our main goal is to contribute some new information on the potential contribution of certain features of dolphins' skulls (in particular, their mandible) to sound localization performance.

\section{EXPERIMENTAL SETUP \& DATA ACQUISITION}

All our experiments are conducted on the skull (cranium and mandible) of a male adult short-beaked common dolphin, shown in Figure 1a. The skull is $\sim 50 \mathrm{~cm}$ long and $\sim 20 \mathrm{~cm}$ wide. The specimen was acquired on loan from the French National Museum of Natural His-

a)

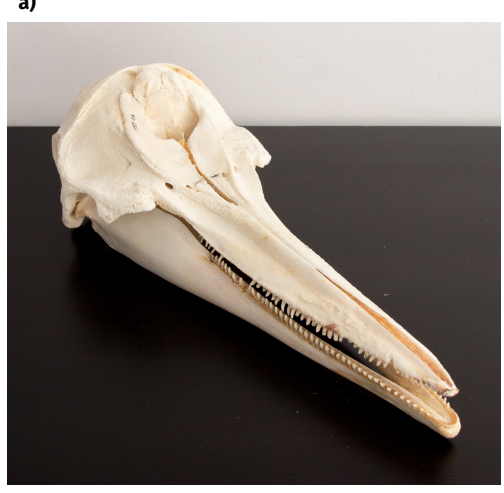

b)

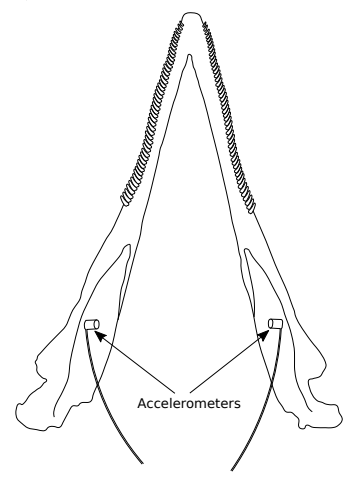

FIG. 1. (Color online) (a) Photograph of the sample (cranium and mandible) used in this study. (b) Sketch of the mandible and the accelerometers glued to it. The accelerometers are approximately $11 \mathrm{~cm}$ apart. 
tory (Muséum National d'Histoire Naturelle, Paris, France), inventory number 1989-06 from the Collection of Comparative Anatomy (Collection d'Anatomie Comparée - Mammifères et Oiseaux). Two miniature piezoelectric charge accelerometers (Brüel $\&$ Kjaer Type 4374) are glued to the inside of the pan bone by a common cyano-acrylate adhesive as shown in Figure 1b. Aroyan (Aroyan, 1996) showed via numerical simulations that acoustic waves entered the head forward of the pan bone, propagated through the outer mandibular fat, to and through the pan bone, continued along intramandibular fats, and converged at the TPC. Measuring the vibration of the pan bone should, therefore, be representative of the sound received at the ears. These sensors weigh $0.75 \mathrm{~g}$ and are characterized by a flat frequency response curve in the frequency range of interest. They are both waterproofed by applying a layer of flexible adhesive. Measurements are conducted in a water tank (6 meters in width, 12 meters in length and 3 meters in depth) filled with chlorinated water kept at the temperature of $\sim 12^{\circ} \mathrm{C}$ throughout the duration of the experiment; the specimen is immersed in the water, centered in depth and in width. Both cranium and mandible are independently suspended and aligned with each other according to the real anatomy. The geometry of the experimental setup is shown in Figure 2. Let us take the midpoint of the segment defined by the accelerometer positions as the origin of a Cartesian reference frame; let the $y$-axis be defined by the accelerometer positions, while the $x$-axis is identified by the tip of the mandible and the origin. The horizontal plane consequently lies roughly on the tooth lines. A broadband marine transducer (Airmar B75L) with an active area of $9.6 \mathrm{~cm}^{2}$ and a transmitting voltage response of around $155 \mathrm{~dB}($ re $1 \mu \mathrm{Pa}$ per volt at $1 \mathrm{~m})$ throughout the used frequency range is placed at a distance of 2 meters away from the origin 


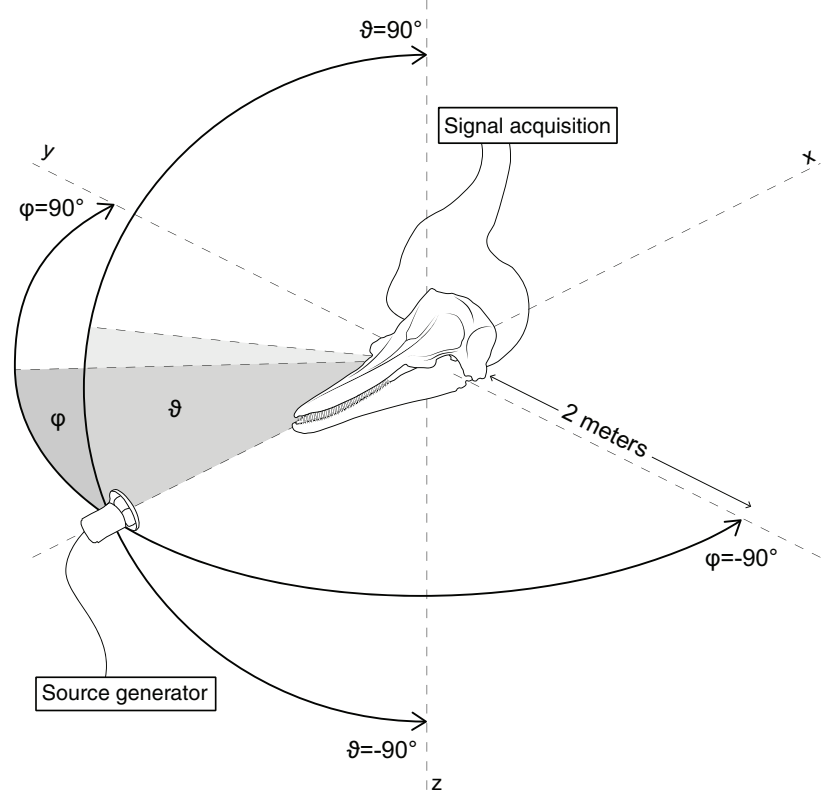

FIG. 2. Sketch of the experimental setup. The sound source moves along two half circles, either in the median or horizontal plane at a distance of $2 \mathrm{~m}$ from the origin.

in front of the skull along the $x$-axis. The skull is then rotated around either the $z$-axis, which corresponds to an angular movement of the transducer in the horizontal plane (i.e. constant source elevation $\vartheta=0^{\circ}$ ), while azimuth $\varphi$ changes from $-90^{\circ}$ nearest the left "ear" to $+90^{\circ}$ closest to the right "ear"), or around the $y$-axis, which corresponds to an angular movement of the transducer in the median plane (i.e. constant $\varphi=0^{\circ}$, while $\vartheta$ changes from $-90^{\circ}$ directly below to $+90^{\circ}$ directly above the origin). Data are recorded first for a discrete set of source azimuths on the skull's horizontal plane, spaced $1^{\circ}$ from one another, from $\varphi=-90^{\circ}$ to $\varphi=+90^{\circ}$, and then for a discrete set of source elevations on the vertical plane, again $1^{\circ}$ from one another, from $\vartheta=-90^{\circ}$ to $\vartheta=+90^{\circ}$. For each source location, the transducer emits two different source signals which are digitally generated through a desktop computer and recorded and processed separately. Each source signal is amplified by $30 \mathrm{~dB}$ through a 
home-made power supply resulting in an emitted sound level of about $185 \mathrm{~dB}$ (re $1 \mu \mathrm{Pa}$ per volt at $1 \mathrm{~m})$.

The source signals are

1. a sinusoidal burst, i.e.

$$
c(t)=\sin \left[\phi_{0}+2 \pi f t\right] w(t),
$$

where $\phi_{0}$ denotes the initial phase of the signal, $f=45 \mathrm{kHz}$ and $w(t)$ is a Tukey window (Harris, 1978), which has a total duration of $100 \mu$ s and tapers the first and the last $15 \mu \mathrm{s}$ of the signals, to ensure their smooth on- and offset;

2. a linear chirp

$$
c(t)=\sin \left[\varphi_{0}+2 \pi\left(f_{0} t+\frac{k}{2} t^{2}\right)\right] w(t),
$$

with minimum frequency $f_{0}=45 \mathrm{kHz}$, maximum frequency $f_{1}=55 \mathrm{kHz}$, chirpyness (i.e., rate of frequency change across the chirp) $k=\frac{f_{1}-f_{0}}{t}$, and $w(t)$ the same Tukey window as above.

The sampling frequency for both signals is $2 \mathrm{MHz}$. The signals and their normalized frequency spectra are shown in Figure 3. Peak frequencies are $45 \mathrm{kHz}$ (sinusoid) an $49 \mathrm{kHz}$ (chirp), and $3 \mathrm{~dB}$ bandwidths are $6 \mathrm{kHz}$ (sinusoid) and $10 \mathrm{kHz}$ (chirp). Since our skull specimen belongs to a short-beaked common dolphin, the duration and peak frequency of source signals are chosen to be in the range of that of echolocation clicks of common dolphins (Richardson et al., 2013; Soldevilla et al., 2008). However, our synthetic signals have a smaller bandwidth since we cannot emit broadband clicks due to the specifications of the equipment. While the sinusoidal burst is used for the investigation of binaural and monau- 

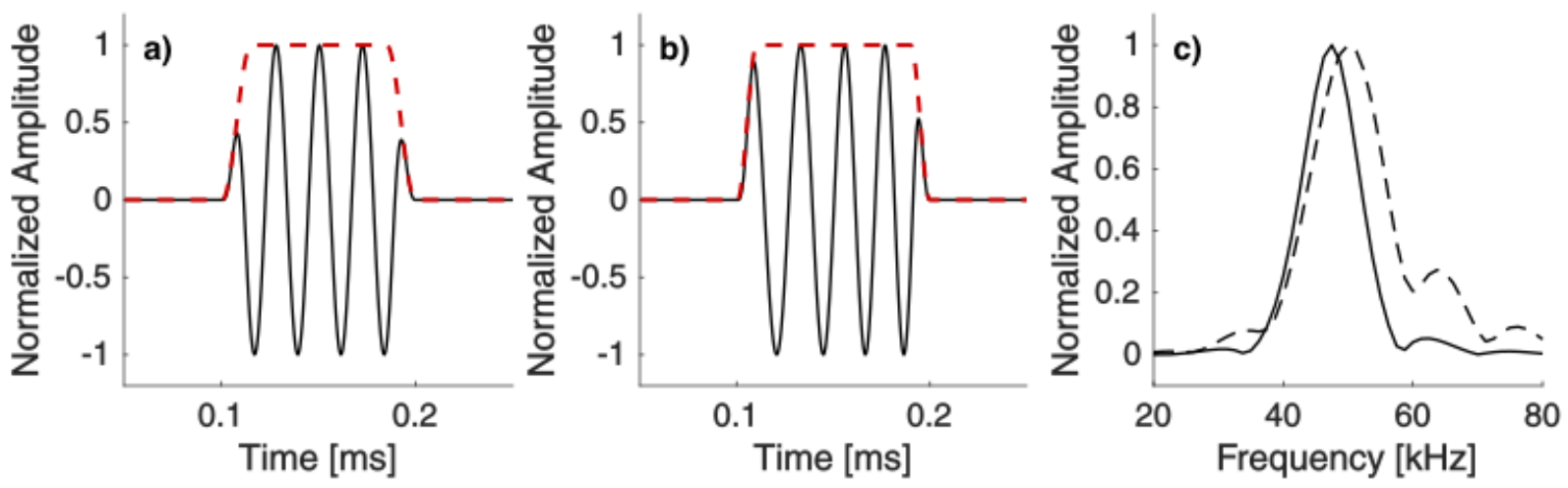

FIG. 3. (Color online) Source signals and their frequency spectra. The Tukey window function used to taper the signals in time is also shown, and is denoted by a dashed red line. a) Sinusoidal burst with a duration of $100 \mu$ s and frequency of $45 \mathrm{kHz}$. b) Narrowband chirp with a duration of $100 \mu \mathrm{s}$ and a frequency range of 45 to $55 \mathrm{kHz}$. c) Normalized spectrum of the sinusoidal burst (solid line) and the chirp (dashed line).

ral cues in both planes, the chirp is solely used for monaural cues. The accelerometers are calibrated to synchronously measure the acceleration of the pan bone on each side of the mandible. At each realization of the experiment, they record for $800 \mu$ s at a sampling rate of $2 \mathrm{MHz}$ using a 16 bit analog-to-digital converter (National Instruments PXIe-6366). The duration of our recordings coincides with the time needed for an acoustic wave to travel $1.2 \mathrm{~m}$ in water, which means that signals reflected from the sides, bottom or surface of the tank are well separated and can be easily identified; we systematically cut our data so that such signals are not taken into account. All recordings are Butterworth bandpass filtered, with cutoff frequencies of $40 \mathrm{kHz}$ and $60 \mathrm{kHz}$ to further reduce unwanted noise.

The entire experiment was repeated three times, including setup and wiring, in order to check consistency and minimize the effect of random errors. All measurements presented 
in the following are obtained by averaging the outcomes of the three experiments, for each combination of source and receiver positions. The associated standard deviation is used as an estimate of measure uncertainty. Throughout this study, we dub "direct" signal the waveform defined by Equations 1 or 2, as it is recorded at the accelerometers after having propagated through water and bone, and being accordingly attenuated. We dub "reverberated" the signal recorded after the direct signal, refracted, reflected, diffracted by and through bone tissue. The so defined reverberated signal is, alone, always longer than $\mathrm{Au}$ et al.'s estimate of $\sim 250 \mu \mathrm{s}$ for a dolphin's integration time (Au et al., 1988). For the sake of simplicity, we neglect reverberations occurring before the end of the direct signal; visual inspection (e.g., Figure 4) shows that their effect is indeed minor, compared to the complex, relatively long coda.

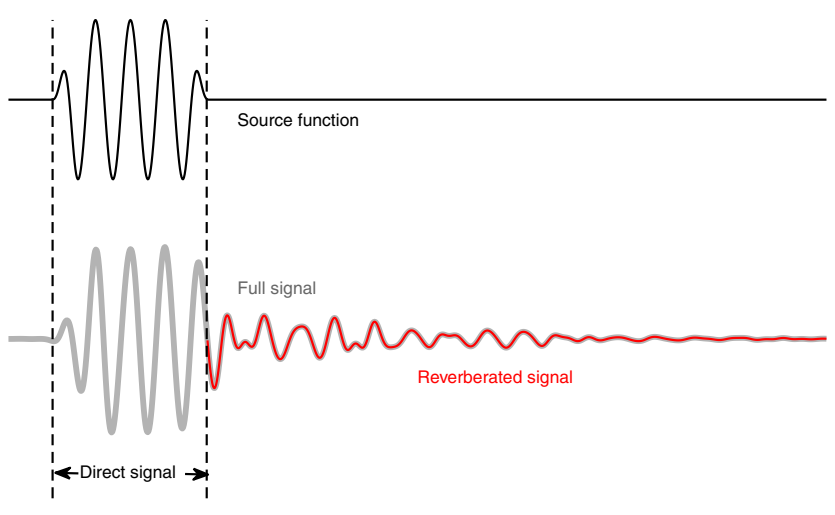

FIG. 4. (Color online) Examples of (top) signal as emitted at the source; (bottom) the same signal, as recorded by one receiver. The recorded trace consists of "direct" (grey) and "reverberated" (red) signals, defined in Section II. 
Reinwald et al., JASA

\section{ITD- AND ILD-BASED SOURCE LOCALIZATION}

We define ITD as the onset time of the direct signal measured at the left accelerometer minus the onset time of the same signal, measured at the right accelerometer. We measure the ITD associated to all our recordings of horizontal- and median-plane sinusoidal sources (Equation 1). This is done by means of a matlab routine that identifies the shape of the source signal in the recorded signal through cross correlation. We show in Fig. 5 the results of this exercise, as functions of source azimuth (if the source is on the horizontal plane) or elevation (if on the vertical plane). For median-plane sources, the ITD should be approximately zero; measured values of ITD accordingly never exceed $6 \mu$ s, corresponding to an error of $0.9 \mathrm{~cm}$ in space. For horizontal-plane sources, by simple geometrical considerations and neglecting HRTF-related diffraction effects (which is reasonable given the absence of soft tissues in our experiment), ITD is expected to approximately coincide with

$$
\operatorname{ITD}(\varphi)=(a / c) \sin (\varphi),
$$

where $a$ is inter-receiver distance and $c$ the speed of sound in water. Again, Figure 5 shows a good agreement between our data and theoretical predictions. Importantly, our measure of ITD should not be taken as an estimate of ITD as perceived by live dolphins, which might be significantly affected by the presence of soft tissues and other anatomical features.

We define ILD as the ratio of the maximum amplitudes (Figure 6) of the direct signal as recorded by left vs. right receivers, in $\mathrm{dB}$, i.e.,

$$
\operatorname{ILD}(\varphi, \vartheta)=20 \log _{10}\left\{\frac{\max \left[s\left(\vartheta, \varphi, \boldsymbol{r}_{L}, t\right)\right]}{\max \left[s\left(\vartheta, \varphi, \boldsymbol{r}_{R}, t\right)\right]}\right\} \quad[\mathrm{dB}]
$$




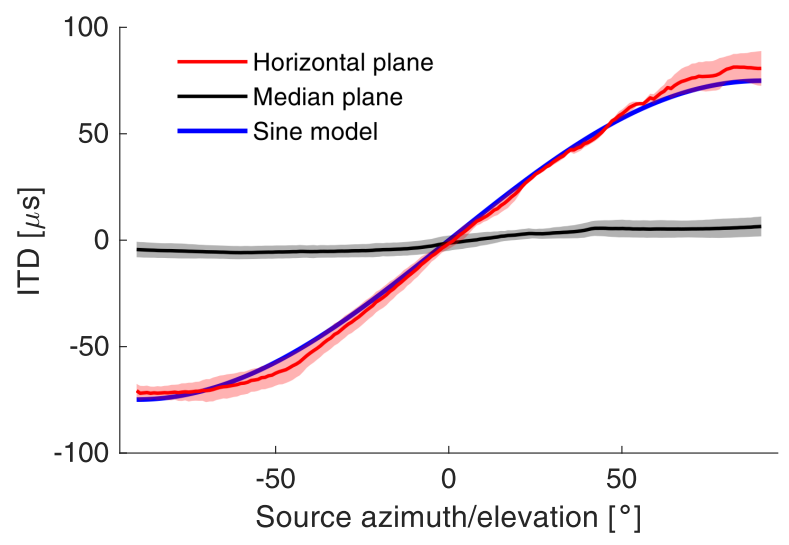

FIG. 5. (Color online) Measured ITD from our binaural recordings of sources deployed on the horizontal plane (red solid line), as a function of source azimuth, and on the vertical plane (black solid line), as a function of source elevation. Color-shaded areas around each solid line denote standard deviation. Expected horizontal-plane ITD based on the theoretical model of Equation 3 is shown as a blue solid line.

where, for the sake of clarity, the signal $s$ is explicitly written as a function of source azimuth and elevation, and receiver position (its only possible values being $\boldsymbol{r}_{L}, \boldsymbol{r}_{R}$ for left and right receiver, respectively). Although other definitions of ILD have been proposed, e.g. in the field of robotics (Youssef et al., 2012), Equation 4 has been used in similar bioacoustic research (Moore and Au, 1975) and can be interpreted similarly to peak values of electrophysiological audiograms (Mulsow et al., 2014; Supin and Popov, 1993). We cannot relate our ILD observations to a simple theoretical model as for the ITD, because of (i) the inherent complexity of waveforms resulting from multiple reverberations within the pan bone, and (ii) our neglect of anatomical features, other than the mandible and skull bones, including cranial air sacks, the albuminous foam (which separates the middle and inner ear from 

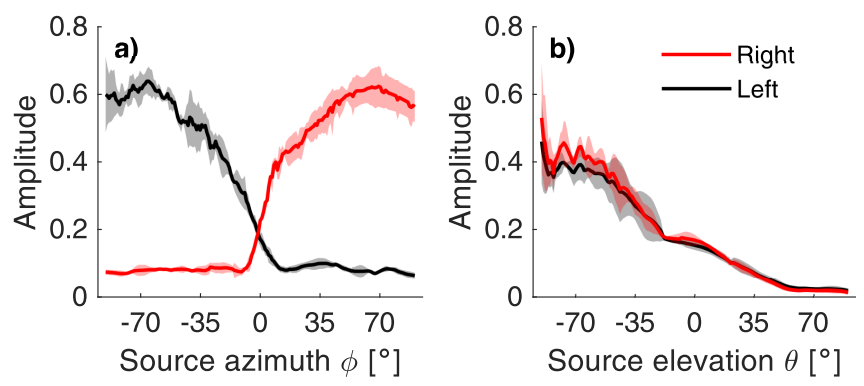

FIG. 6. (Color online) Maximum amplitudes recorded at the left (black) and right (red) receivers of sources deployed on (a) the horizontal plane, as a function of source azimuth, and (b) the vertical plane, as a function of source elevation. Color-shaded areas around each solid line denote standard deviation.

the skull) and acoustically functional fats, that are likely to contribute to ILD (Ketten, 1992; Supin and Popov, 1993) and, interestingly, introduce significant dispersion (Aroyan, 2001). Also, because our setup does not account for such complexity, our data cannot be directly compared to experimental data or realistic numerical ILD models. Figure 7 shows our measures of ILD, derived from waveform data via Equation 4, as a function of source azimuth and elevation.

As expected, ILD values associated with median-plane sources are close to 0 , with fluctuations of less than $2 \mathrm{~dB}$. For horizontal-plane sources, the ILD ranges between $18 \mathrm{~dB}$ and $-18 \mathrm{~dB}$, changing most rapidly directly in front of the dolphin's beak, at $\varphi$ between $-10^{\circ}$ and $10^{\circ}$. In this range of $\varphi$, ILD decreases from $13 \mathrm{~dB}$ down to $-12 \mathrm{~dB}$, losing more than $1 \mathrm{~dB}$ per degree. This is an effect of sound shadowing by bone tissues, as the receiver at $x_{L}$ loses direct acoustic sight of the sound source when this is rotated to the opposite side of the 
Reinwald et al., JASA

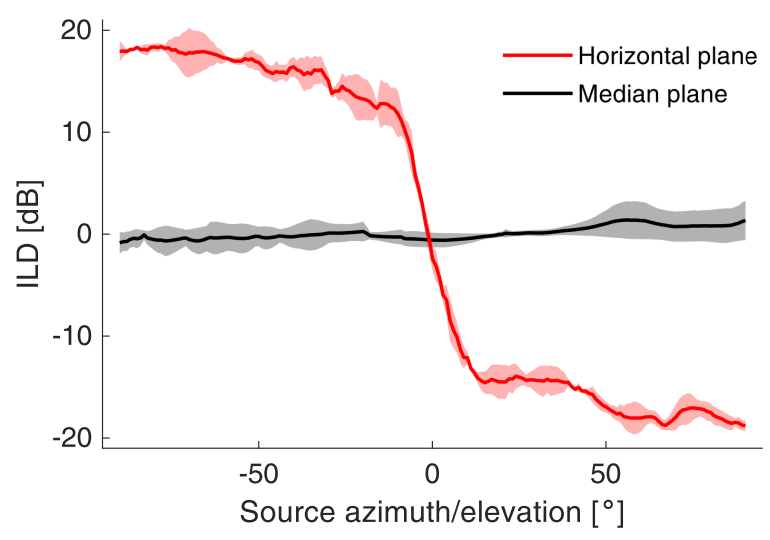

FIG. 7. (Color online) ILD in both planes. Mean ILD (solid lines) and their standard deviation (color shaded areas) of three independent measurements are shown in red (horizontal plane) and black (median plane).

mandible. At larger, positive or negative, azimuths, the ILD grows less rapidly, at a rate of less than $1 \mathrm{~dB}$ per degree, and fluctuations (standard deviation) up to $\pm 2 \mathrm{~dB}$.

The results in Figures 5 through 7 are not new or surprising per se, but confirm some simple, well known properties of all binaural auditory systems. Importantly, the left-right symmetries of our data and the fit between data and a simple ITD model confirm that our setup is correct, and adequate to the applications that follow.

\section{CORRELATION-BASED SOURCE LOCALIZATION}

Waves that interact with a complex HRTF carry a great wealth of information, that could in principle be exploited to localize their sources. Both binaural and monaural cues discussed so far only exploit a small portion of such information. While it has been established that humans and other terrestrial species localize via those cues alone, the echolocation performance 
observed in dolphins suggests that their auditory system might include a more sophisticated localization mechanism. We implement a simple algorithm to localize sources, based on the time-reversal concept developed by Mathias Fink and co-workers (e.g., Catheline et al., 2007; Fink et al., 2000).

\section{A. Accuracy of source localization by correlation}

We conduct a "time-reversal" exercise based on the theoretical formulation developed in the appendix. Specifically, we implement the right-hand side of Equation A.5 and study its effectiveness as a source-localization algorithm. As explained in detail in the appendix, in the context of echolocation "time reversal" as defined e.g. by Catheline et al. (Catheline et al., 2007) is equivalent to a simple correlation of each newly perceived signal with a library of echoes previously heard and "stored." Accordingly, pairs of traces $s\left(\mathbf{r}_{R}, \mathbf{r}_{A}, t\right), s\left(\mathbf{r}_{R}, \mathbf{r}_{B}, t\right)$ recorded at $\mathbf{r}_{R}$ as described in Section II, are cross-correlated to one another, for all possible pairs of source locations $\mathbf{r}_{A}, \mathbf{r}_{B}$. The same is done for traces recorded at $\mathbf{r}_{L}$. As a result, for each source location $\mathbf{r}_{B}$, we obtain the correlation between the corresponding recorded signal and the signal associated to all other possible sources $\left(\mathbf{r}_{A}\right)$. Because it is closely related to how sharply a time-reversed wave field would focus at $\mathbf{r}_{B}$ (see appendix), we dub it "focusing function". Since, in this study, we are looking at sources on the horizontal and median planes only, the focusing function depends on either $\vartheta$ or $\varphi$ only; by definition, it is exactly 1 when both $\vartheta$ and $\varphi$ are the same as those of the actual source.

For the sake of simplicity (and speed), cross correlation is implemented by first shifting each pair of signals to have zero lag, and then calculating the correlation between the shifted 
traces. Intensity differences between the two correlated signals are also irrelevant, as the convolution product is normalized so that the auto-correlation at zero lag equals 1.

We next visualize how well a source is localized by our algorithm as a function of its true location. This is shown in Figure 8 through 11 where the horizontal and vertical axes of each plot correspond to the azimuth $\varphi_{0}$ or elevation $\vartheta_{0}$ of the true source and of all recorded sources $\left(\varphi_{i}, \vartheta_{i}\right)$. Specifically, focusing functions obtained based on the chirp-like source in the median plane are plotted in Figure 8, while Figure 9 shows the corresponding results for the sinusoidal source. By definition, values on the diagonal of all panels in both figures are 1; near the diagonal, correlations decrease monotonously in all panels; some relevant fluctuations are then observed in both figures for $\vartheta_{i}$ far from $\vartheta_{0}$ when both direct and reverberated signals are correlated, but not when the reverberated signal alone is considered. In the latter case, the focusing function is much sharper, particularly in the $-50^{\circ}$ to $20^{\circ}$ elevation range, and its sharpness does not seem to depend on source elevation $\vartheta_{0}$.

To study how the resolution of our algorithm depends on the true source position in the median plane, we visualize (Figure 10a for the chirp-like source function, Figure 10b for the sinusoidal source function) the increment in $\vartheta$ needed for the focusing function to decrease to $70 \%$ of its maximum, i.e. the $-3 \mathrm{~dB}$ width of the focusing function, which is a rule-of-thumb criterion frequently used in time-reversal acoustics (Catheline et al., 2007; Ing et al., 2005; Kim et al., 2003). The smaller the value of the $-3 \mathrm{~dB}$ width, the higher the resolution, and the performance that can be expected in identifying the true source location. The value of $3 \mathrm{~dB}$ is of no particular physical or biological significance: it is only chosen in analogy with the mentioned studies. This is adequate to our goals, as we are not attempting to 


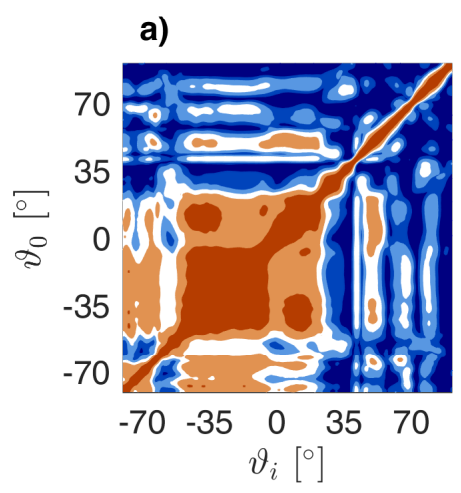

d)

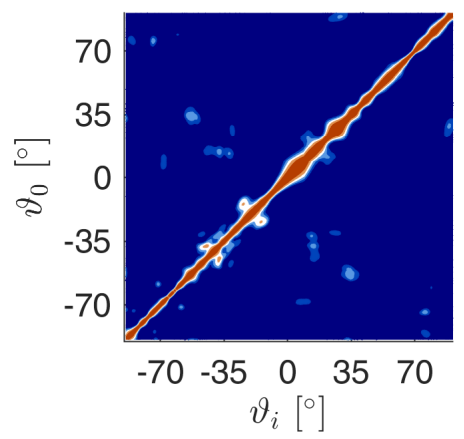

b)

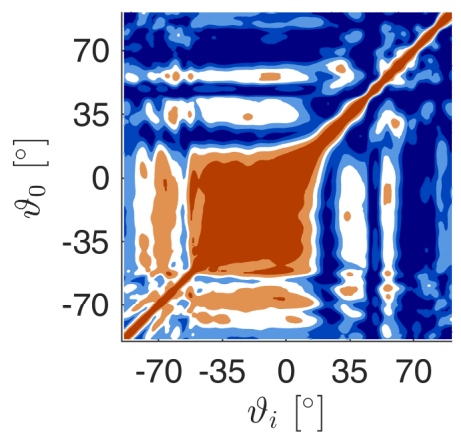

e)

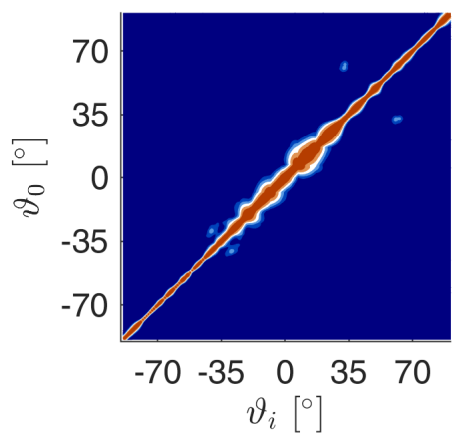

c)

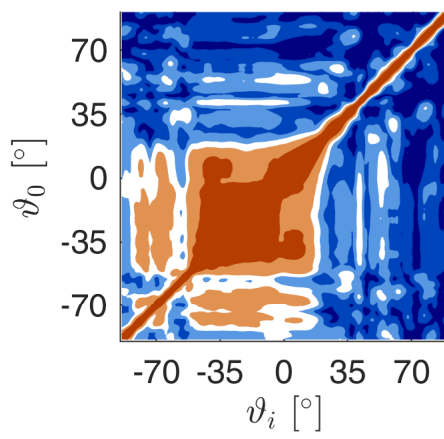

f)

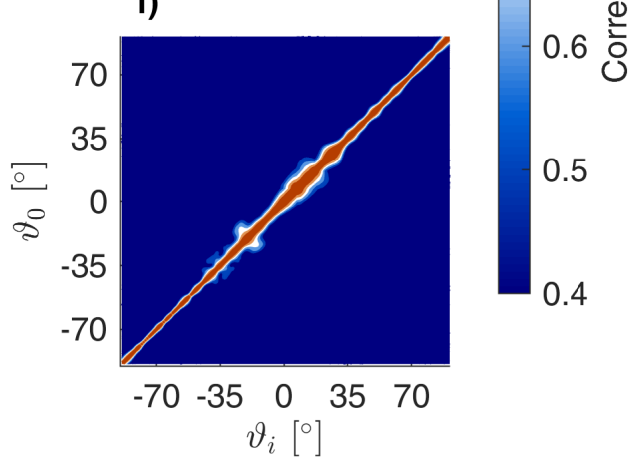

FIG. 8. (Color online) Focusing functions in the median plane using the chirp-like source function as determined from the entire waveform, recorded by the (a) left, (b) right, and (c) both (sum of (a) and (b)) accelerometers, and from the reverberated waveform alone, again at (d) left, (e) right, and (f) both accelerometers. Each row of a given panel shows, accordingly, the maximum cross correlation value between the signal associated with one particular source (defined by its elevation $\left.\vartheta_{0}\right)$, and those of all other sources (elevations $\vartheta_{i}$ on the horizontal axis).

reproduce absolute, observed MAA values, but rather to estimate the relative changes in the resolution in source localization. Figures 8 through 10 show that direct signal alone does not provide sufficient information to discriminate sources in the $-50^{\circ}$ to $20^{\circ}$ elevation range; on the contrary, it obscures the information contained in the reverberated signal, which, 


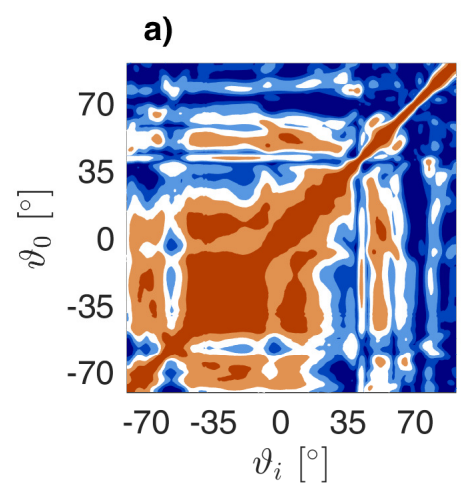

d)

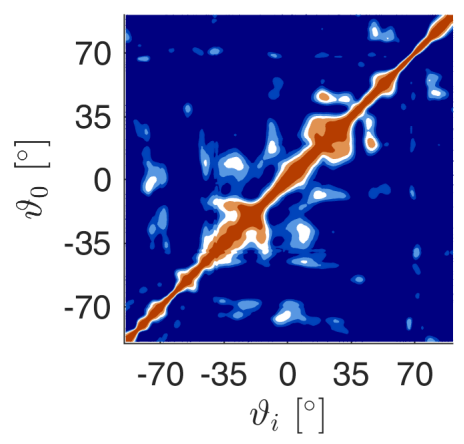

b)

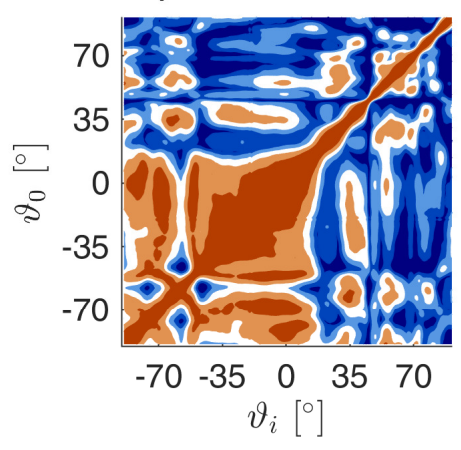

e)

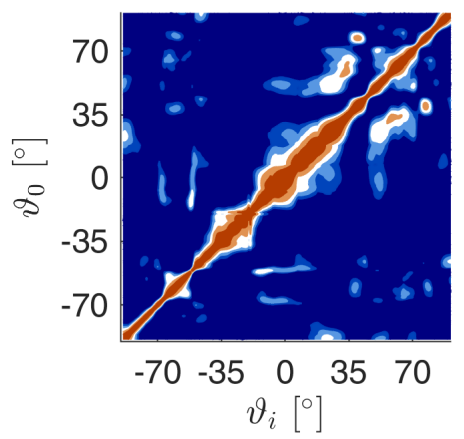

c)

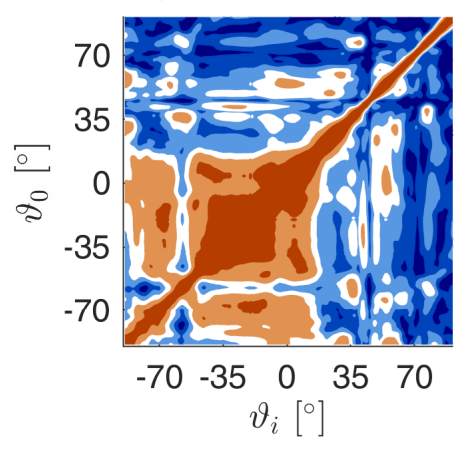

f)

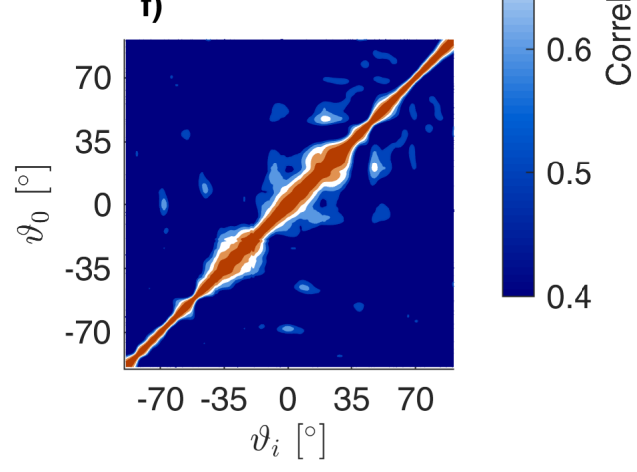

FIG. 9. (Color online) Focusing functions in the median plane using the sinusoidal source function.

Panels are structured the same way as in Figure 8.

if used by itself, actually results in much sharper focusing functions. It is apparent from our results that our algorithm achieves approximately equal accuracy for monochromatic vs multi-frequency signals (Figure $8 \& 9$ ). Figure 10 shows that localization of a sinusoidal source affords slightly lower resolution (larger $-3 \mathrm{~dB}$ widths) throughout all elevations. As to be expected, widening the frequency band of the source increases the resolution of this algorithm. Similar inferences can be made based on the focusing functions obtained from horizontal-plane sources, which are shown in Figure 11. In this case, the resolution highly benefits from analyzing the reverberated signal alone, if the source is on the same side of the 

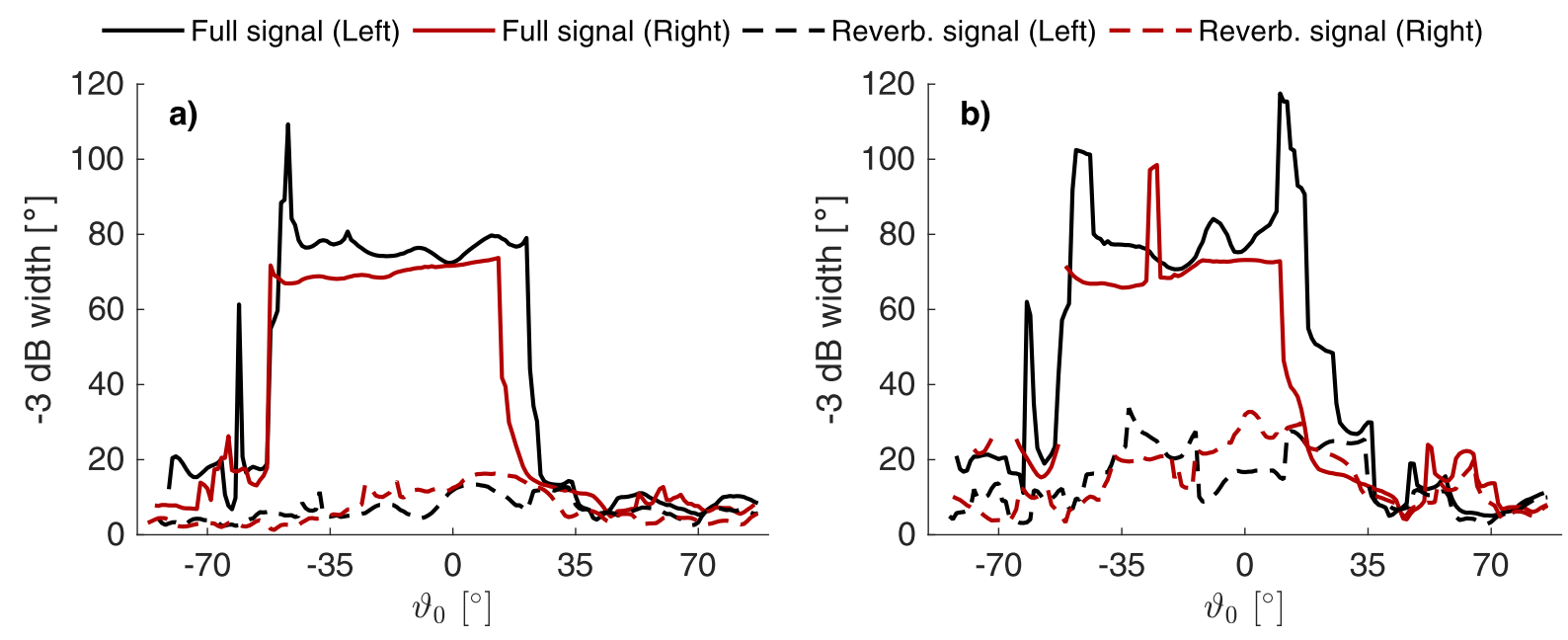

FIG. 10. (Color online) $-3 \mathrm{~dB}$ widths of the focusing functions in the median plane using a) the chirp-like source function and b) the sinusoidal source function.

skull as the respective receiver. Interestingly, the $-3 \mathrm{~dB}$ width is similar to that extrapolated from Figures $8 \& 9$, i.e. our algorithm is about equally sensitive to changes in azimuth vs elevation of the source.

\section{SUMMARY AND CONCLUSIONS}

We have developed a source localization algorithm (Section IV) based on the cross correlation of an observed signal with a library of known signals, each corresponding to a different source location. We have implemented the algorithm in the context of a biosonar application (Equation A.5 and related discussion), and "source" should be interpreted here as synonymous with biosonar "target" (or "secondary" source). We have substantiated our source-localization metric from a theoretical standpoint, by drawing an analogy between cross correlation and the theory of acoustic time reversal. We have evaluated the 
a)

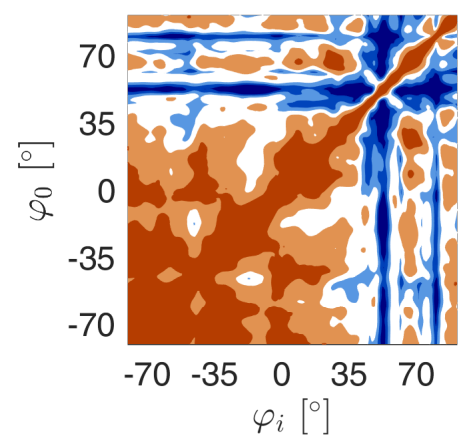

d)

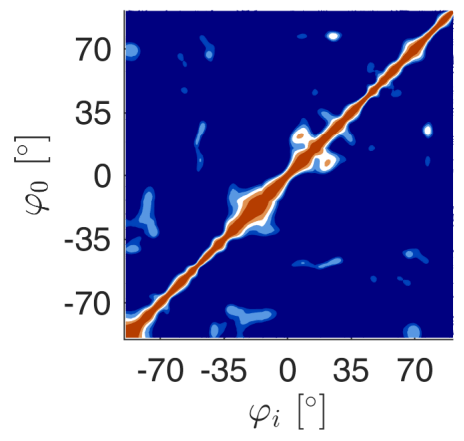

b)

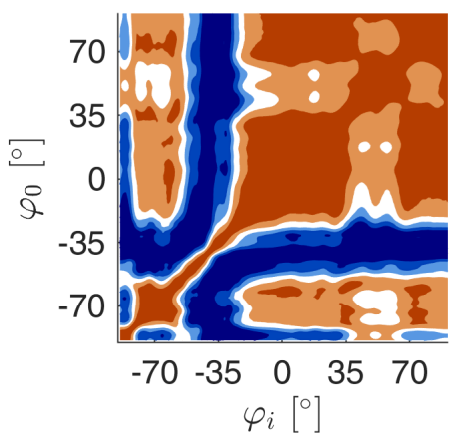

e)

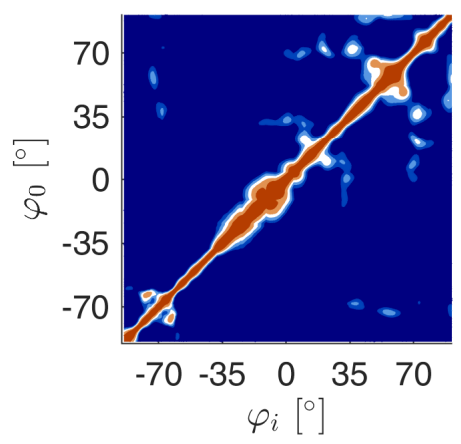

c)

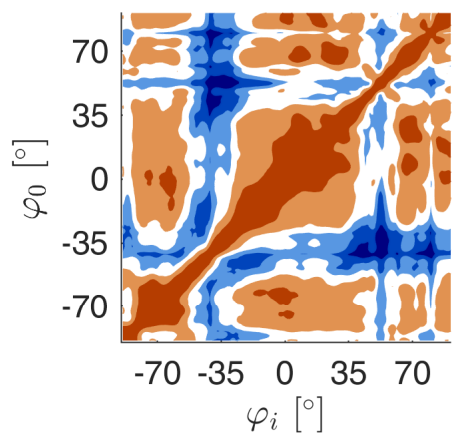

f)

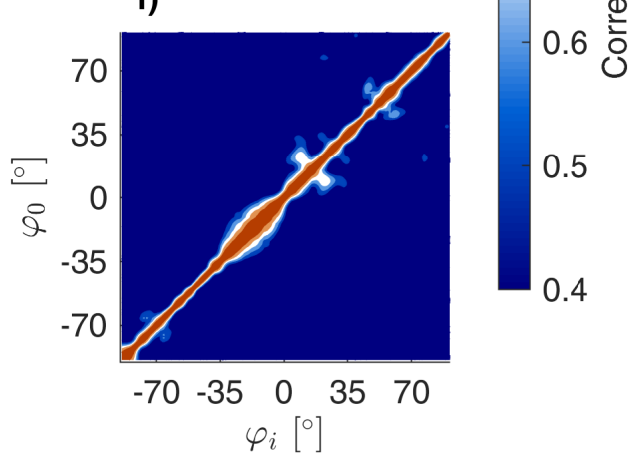

We quantify the performance of our algorithm via the width of the the focusing function, or, in other words, the rate at which correlation decreases, as an observed signal is compared with library signals associated with sources increasingly far from the true one. We find that this width is significantly reduced (the rate of correlation loss is accelerated) when 
the direct signal, which is simply an attenuated version of the original chirp/sinusoidal burst, is subtracted from the recorded waveform before cross correlation. This way, only the reverberated coda, most strongly affected by the shape and properties of the skull, is actually employed in localization: localizing by reverberated signal alone (rather than the entire wavetrain) sharpens source resolution.

The spatial accuracy of source localization by dolphins has been observed, through behavioral experiments, to be equally accurate independent of source azimuth and elevation, i.e., it has approximately constant resolution over the entire solid angle (Nachtigall, 2016). This property of dolphins is counter-intuitive, if one considers that humans and other species have presumably evolved pinnae to help determine the elevation of sound sources (Section I), while cetaceans have actually lost them. We infer that, to achieve such performance, the dolphin's auditory system might make use of a unique, and currently unknown localization tool, particularly effective for sources in the median plane or along the "cone of confusion." Our results do not directly constrain the nature of the sound localization system implemented in a dolphin's brain; yet, they do show that signal reverberated within the dolphin's skull (with the mandible playing the most prominent role) varies significantly as a function of (that is to say, is very sensitive to) source location. The "direct" signal, i.e. signal without reverberation, appears to be much less sensitive to source location. We have shown that reverberated signal contains sufficient information to discriminate median-plane sources, and that this could be achieved by simply cross-correlating any newly perceived sound with a library of previously recorded data. 
While our model shares with dolphins some relevant features, we are hardly reproducing the signals that would be perceived by actual, live specimens. Importantly, we conduct our experiments on the skull alone, neglecting the effects of muscles, fats, and other soft tissues. While we plan to surpass this limitation in our future work, at this point we consider it to be partly justified, first, by the fact that acoustic waves propagate through soft tissues at about the same speed as through water (e.g., Gray and Rogers, 2017; Soldevilla et al., 2005), which limits wave-propagation effects. Mandible and skull, on the contrary, provide a strong wave speed contrast (a factor of about two) resulting in significant diffraction, reverberation, etc. Cranford et al. show that the amplitude of perceived signals is significantly affected by the anatomical features of soft tissues in the head (Cranford et al., 2008), but we speculate that phase, more than amplitude, is relevant to source localization (correlation being mostly sensitive to phase). Secondly, the neglect of soft tissues allows us to isolate the specific effects of bone-conducted waves, before additional experiments are conducted on whole heads. In future studies, the issue of a dolphin's resolution of the fine features of acoustic signals with respect to time will also need to be addressed, taking into account e.g. the concept of cochlear integration time as defined by Au et al. (Au et al., 1988). Finally, we envisage to extend our analysis to a broader frequency range, whether by numerical modeling, or new experiments relying on better, currently unavailable hardware.

In summary, our results indicate that, within a good approximation, a one-to-one correspondence exists between the waveform of the bone-conducted, reverberated coda as recorded at a dolphin's ear locations, and the locations of the source (or, in principle, the reflecting target) that originally generated (or reflected) the signal. While we have no 
436

437

knowledge of how such information might be processed and exploited by the brain, we speculate that bone-conducted, reverberated sound could contribute to explaining the peculiar, poorly understood accuracy of sound localization in odontocete cetaceans.

\section{ACKNOWLEDGMENTS}

This project has received funding from the European Union's Horizon 2020 research and innovation programme under the Marie Sklodowska-Curie grant agreement No 641943 (ITN WAVES). We are grateful to Christine Lefèvre, and to the Collection of Comparative Anatomy at the French National Museum of Natural History, for providing the skull specimen on which all our experiments were conducted. We are likewise grateful to the editor W. W. L. Au and two anonymous reviewers, for their insightful comments to our manuscript. 


\section{APPENDIX: TIME-REVERSAL THEORY AND CROSS CORRELATION}

It is well known that, if attenuation is neglected, the imaginary part $(\Im)$ of the acoustic Green's function (i.e., impulse response) $G$ associated with a source at $\mathbf{r}_{A}$ and a receiver at $\mathbf{r}_{B}$ (or vice-versa) can be obtained by the frequency-domain relationship

$$
\frac{\rho c}{\omega} \Im\left[G\left(\mathbf{r}_{A}, \mathbf{r}_{B}\right)\right]=-\int_{\partial V} \mathrm{~d}^{2} \mathbf{r}\left[G^{*}\left(\mathbf{r}, \mathbf{r}_{B}\right) G\left(\mathbf{r}, \mathbf{r}_{A}\right)\right]
$$

(e.g., Boschi and Weemstra, 2015, Equation (103)), where $G$ is the 3-D Green's function, $\partial V$ is an arbitrary closed surface surrounding $\mathbf{r}_{A}$ and $\mathbf{r}_{B}$, and $\rho, c, \omega$ denote density, speed of sound and frequency, respectively. * stands for complex conjugation, so that the integrand at the right-hand side of Equation A.1 is the Fourier transform of the time-domain cross correlation of $G\left(\mathbf{r}, \mathbf{r}_{B}, t\right)$ and $G\left(\mathbf{r}, \mathbf{r}_{A}, t\right)$.

Think now of $\mathbf{r}_{B}$ as the location of an acoustic source (e.g., Boschi and Weemstra, 2015); $G\left(\mathbf{r}, \mathbf{r}_{B}, \omega\right)$ is the Fourier-transform of an impulse generated at $\mathbf{r}_{B}$ and recorded by a receiver at $\mathbf{r} ; G^{*}\left(\mathbf{r}, \mathbf{r}_{B}, \omega\right)$ is the Fourier transform of the same signal, reversed in time. Imagine that the time-reversed signal be then emitted from $\mathbf{r}$ and recorded at another point $\mathbf{r}_{A}$ : this amounts to convolving (in the frequency domain, multiplying) the time-reversed signal with the Green's function $G\left(\mathbf{r}_{A}, \mathbf{r}, \omega\right)$. Eq. (A.1) then shows that by repeating time reversal and propagation ("backward in time") for all points $\mathbf{r}$ on $\partial V$, and summing all the resulting traces at $\mathbf{r}_{B}$, the imaginary part of the Green's function between $\mathbf{r}_{B}$ and $\mathbf{r}_{A}$ is obtained. Note that the imaginary part of the frequency-domain $G$ coincides, in the time domain, with the inverse Fourier transform

$$
F^{-1}\left\{\Im\left[G\left(\mathbf{r}_{A}, \mathbf{r}_{B}, \omega\right)\right]\right\}=G\left(\mathbf{r}_{A}, \mathbf{r}_{B},-t\right)-G\left(\mathbf{r}_{A}, \mathbf{r}_{B}, t\right)
$$


i.e., as $t$ grows from $-\infty$ to 0 , a time-reversed Green's function, followed by a regular $G$ with its sign reversed (e.g. Fink, 2006).

It is inferred that the Green's function between $\mathbf{r}_{A}$ and $\mathbf{r}_{B}$ can be reconstructed from an impulse emitted at $\mathbf{r}_{B}$ and recorded at a set of points $\mathbf{r}$ that sample $\partial V$, by (i) time-reversing the signal $G\left(\mathbf{r}, \mathbf{r}_{B}, \omega\right)$ emitted by $\mathbf{r}_{B}$ and recorded at $\mathbf{r}$; (ii) convolving the time-reversed signal $G^{*}\left(\mathbf{r}^{\prime}, \mathbf{r}_{B}, \omega\right)$ with the impulse response $G\left(\mathbf{r}_{A}, \mathbf{r}, \omega\right)$ between $\mathbf{r}$ and $\mathbf{r}_{A}$; (iii) iterating over all receivers $\mathbf{r}$; (iv) summing the resulting signals. This procedure is usually referred to as "acoustic time reversal," because the wave field so obtained is essentially a time-reversed, backward propagated version of the original impulse response $G$ (Fink, 2006); as such, it will naturally focus at the original source location, where it will show a very prominent maximum. An important consequence of this is that time reversal can be used as a source localization tool: if a signal generated by a source at an unknown location $\mathbf{r}_{B}$ is recorded by an array of receivers forming a closed surface $\partial V$, by implementing numerically steps (i) through (iv) above and looking for the maximum of the resulting numerical wave field, the source location can be determined.

While in principle $G$ is accurately reconstructed (the time-reversed wave field focuses at the original source location) only if recordings made at a dense, uniform array of receivers are time-reversed and backward propagated, many studies have shown that focusing can also be achieved using a much smaller receiver array, provided that the medium of propagation has some relevant 3-D structure, and that this structure is known and properly accounted for when modeling wave propagation. In particular, it was shown (Catheline et al., 2007) that a pair of receivers, deployed at ear locations on a human skull, are enough for the 
time-reversed, backward-propagated signal to sharply focus at the source; since our setup is essentially the same, we can reasonably expect two-receiver time reversal to perform about equally well in our case. Equation A.1 can thus be simplified to

$$
\Im\left[G\left(\mathbf{r}_{A}, \mathbf{r}_{B}\right)\right] \propto G^{*}\left(\mathbf{r}_{L}, \mathbf{r}_{B}\right) G\left(\mathbf{r}_{L}, \mathbf{r}_{A}\right)+G^{*}\left(\mathbf{r}_{R}, \mathbf{r}_{B}\right) G\left(\mathbf{r}_{R}, \mathbf{r}_{A}\right)
$$

where, for the sake of simplicity, and since we are only interested in finding the maxima of the expressions in question, we have dropped absolute amplitude information. Equation A.3 is only valid for impulsive signals, but it is straightforward to generalize it to an arbitrary signal $s(\omega)$. Write $s$ as the convolution $s\left(\mathbf{x}_{1}, \mathbf{x}_{2}, \omega\right)=h(\omega) G\left(\omega, \mathbf{x}_{1}, \mathbf{x}_{2}\right)$, with $h$ an arbitrary "source time function" independent of the source and receiver positions $\mathbf{x}_{1}$ and $\mathbf{x}_{2}$. If one multiplies both sides of Equation A.3 by $h^{*}(\omega)$,

$$
h^{*}(\omega) \Im\left[G\left(\mathbf{r}_{A}, \mathbf{r}_{B}\right)\right] \propto s^{*}\left(\mathbf{r}_{L}, \mathbf{r}_{B}\right) G\left(\mathbf{r}_{L}, \mathbf{r}_{A}\right)+s^{*}\left(\mathbf{r}_{R}, \mathbf{r}_{B}\right) G\left(\mathbf{r}_{R}, \mathbf{r}_{A}\right)
$$

The convolution of $s^{*}$ with $G$ at the right-hand side of Equation A.4 should be interpreted, again, as backward propagation of the time-reversed recorded signal s; Equation A.4 stipulates that, by this procedure (in the assumption that sufficient information about the wave field be recorded by a pair of receivers alone), a source of arbitrary complexity (with respect to time) can be reconstructed: the time-reversed signal will focus at the source, where a receiver would approximately record the original source time function $h(t)$, reversed in time.

We take here a slightly different approach (Catheline et al., 2007). Let us multiply both sides of Equation A.3 by $|h(\omega)|^{2}$,

$$
|h(\omega)|^{2} \Im\left[G\left(\mathbf{r}_{A}, \mathbf{r}_{B}\right)\right] \propto s^{*}\left(\mathbf{r}_{L}, \mathbf{r}_{B}\right) s\left(\mathbf{r}_{L}, \mathbf{r}_{A}\right)+s^{*}\left(\mathbf{r}_{R}, \mathbf{r}_{B}\right) s\left(\mathbf{r}_{R}, \mathbf{r}_{A}\right)
$$


Note that the products at the right-hand side of Equation A.5 can be interpreted, in the time domain, as both the convolution of $s\left(\mathbf{r}_{L, R}, \mathbf{r}_{A}, t\right)$ with the time-reversed counterpart of $s\left(\mathbf{r}_{L, R}, \mathbf{r}_{B}, t\right)$, and the cross correlation of $s\left(\mathbf{r}_{L, R}, \mathbf{r}_{A}, t\right)$ and $s\left(\mathbf{r}_{L, R}, \mathbf{r}_{B}, t\right)$ (Derode et al., 2003; Draeger and Fink, 1999). As opposed to Equation A.4, the right-hand side of Equation A.5 does not allow one to reconstruct, from the data, the signal as originally emitted at $\mathbf{r}_{B}$ (because $\left(s \mathbf{r}_{R, L}, \mathbf{r}_{A}\right)$ are unknown and cannot be computed). Equation A.5 can be relevant, however, if the time function $h(t)$ is known, while the location of the source is to be determined. This applies, for instance, to echolocating species, that identify and analyze echoes of signals that they have themselves emitted. Echolocation can presumably be learned by training, which is equivalent to forming a "library" of observed echoes $s\left(\mathbf{r}_{R, L}, \mathbf{r}_{A}\right)$ associated with a given emitted signal and known target locations $\mathbf{r}_{A}$ : each time a relevant signal is perceived, the echolocating agent would then systematically compare it to all recorded traces $s\left(\mathbf{r}_{R, L}, \mathbf{r}_{A}\right)$, each corresponding to a different value of $\mathbf{r}_{A}$ eventually covering the entire solid angle. Imagine that this comparison be implemented via cross correlation: this is equivalent to implementing the right-hand side of Equation A.5, and the same equation implies that cross correlation should be maximum when $\mathbf{r}_{A}=\mathbf{r}_{B}$; the sharpness of focusing at the source, and thus the accuracy of source localization, is strictly related to how well a timereversed, backward propagated wave field would focus at the original source. Importantly, however, the proposed algorithm does not involve any wave propagation modeling, but is based entirely on signal processing of measurements at two receivers. 
Agterberg, M. J., Snik, A. F., Hol, M. K., van Esch, T. E., Cremers, C. W., Van Wanrooij, M. M., and Van Opstal, A. J. (2011). "Improved horizontal directional hearing in bone conduction device users with acquired unilateral conductive hearing loss," Journal of the Association for Research in Otolaryngology 12(1), 1-11.

Aroyan, J. L. (1996). Three-dimensional numerical simulation of biosonar signal emission and reception in the common dolphin (University of California, Santa Cruz).

Aroyan, J. L. (2001). "Three-dimensional modeling of hearing in delphinus delphis," The Journal of the Acoustical Society of America 110(6), 3305-3318.

Aroyan, J. L., Cranford, T. W., Kent, J., and Norris, K. S. (1992). "Computer modeling of acoustic beam formation in d elphinusdelphis," The Journal of the Acoustical Society of America 92(5), 2539-2545.

Au, W. W. (2012). The sonar of dolphins (Springer Science \& Business Media).

$\mathrm{Au}$, W. W., and Fay, R. R. (2012). Hearing by whales and dolphins, 12 (Springer Science \& Business Media).

Au, W. W., and Hastings, M. C. (2008). Principles of marine bioacoustics (Springer).

Au, W. W., Moore, P. W., and Pawloski, D. A. (1988). "Detection of complex echoes in noise by an echolocating dolphin," The Journal of the Acoustical Society of America 83(2), $662-668$.

Batteau, D. W. (1967). "The role of the pinna in human localization," Proc. R. Soc. Lond. B 168(1011), 158-180. 
Blauert, J. (1969). "Sound localization in the median plane," Acta Acustica united with Acustica 22(4), 205-213.

Blauert, J. (1997). Spatial hearing: the psychophysics of human sound localization (MIT press).

Boschi, L., and Weemstra, C. (2015). "Stationary-phase integrals in the cross correlation of ambient noise," Reviews of Geophysics 53(2), 411-451.

Branstetter, B. K., and Mercado III, E. (2006). "Sound localization by cetaceans," International Journal of Comparative Psychology 19(1).

Brill, R. L., Moore, P. W., and Dankiewicz, L. A. (2001). "Assessment of dolphin (tursiops truncatus) auditory sensitivity and hearing loss using jawphones," The Journal of the Acoustical Society of America 109(4), 1717-1722.

Brill, R. L., Sevenich, M. L., Sullivan, T. J., Sustman, J. D., and Witt, R. E. (1988). "Behavioral evidence for hearing through the lower jaw by an echolocating dolphin (tursiops truncatus)," Marine Mammal Science 4(3), 223-230.

Butler, R. A., and Belendiuk, K. (1969). "Monaural and binaural localization of noise bursts vertically in the median sagittal plane," Journal of the Auditory Research 3, 230-235.

Butler, R. A., Humanski, R. A., and Musicant, A. D. (1990). "Binaural and monaural localization of sound in two-dimensional space," Perception 19(2), 241-256.

Catheline, S., Fink, M., Quieffin, N., and Ing, R. K. (2007). "Acoustic source localization model using in-skull reverberation and time reversal," Applied physics letters 90(6), 063902. 
Cranford, T. W., and Krysl, P. (2015). "Fin whale sound reception mechanisms: skull vibration enables low-frequency hearing," PloS one 10(1), e0116222.

Cranford, T. W., Krysl, P., and Hildebrand, J. A. (2008). "Acoustic pathways revealed: simulated sound transmission and reception in cuvier's beaked whale (ziphius cavirostris)," Bioinspiration \& Biomimetics 3(1), 016001.

Derode, A., Larose, E., Tanter, M., De Rosny, J., Tourin, A., Campillo, M., and Fink, M. (2003). "Recovering the greens function from field-field correlations in an open scattering medium (1)," The Journal of the Acoustical Society of America 113(6), 2973-2976.

Dible, S. A., Flint, J. A., and Lepper, P. A. (2009). "On the role of periodic structures in the lower jaw of the atlantic bottlenose dolphin (tursiops truncatus)," Bioinspiration \& biomimetics 4(1), 015005.

Draeger, C., and Fink, M. (1999). "One-channel time-reversal in chaotic cavities: Theoretical limits," The Journal of the Acoustical Society of America 105(2), 611-617.

Fink, M. (2006). "Time-reversal acoustics in complex environments," Geophysics 71(4), SI151-SI164.

Fink, M., Cassereau, D., Derode, A., Prada, C., Roux, P., Tanter, M., Thomas, J.-L., and Wu, F. (2000). "Time-reversed acoustics," Reports on Progress in Physics 63(12), 1933.

Graf, S., Blondel, P., Megill, W. M., and Clift, S. E. (2009). "Acoustic modelling of dolphin sound reception and implications for biosonar design," in OCEANS 2009-EUROPE, IEEE, pp. $1-6$.

Gray, M. D., and Rogers, P. H. (2017). "In vivo ultrasonic attenuation in cetacean soft tissues," The Journal of the Acoustical Society of America 141(2), EL83-EL88. 
Harris, F. J. (1978). "On the use of windows for harmonic analysis with the discrete fourier transform," Proceedings of the IEEE 66(1), 51-83.

Hartmann, W. M. (1999). "How we localize sound," Physics today 52, 24-29.

Heffner, H. E., and Heffner, R. S. (2016). "The evolution of mammalian sound localization," Acoustics Today 12, 20-27.

Heffner, R. S., and Heffner, H. E. (1992). "Evolution of sound localization in mammals," in The evolutionary biology of hearing (Springer), pp. 691-715.

Hunter, J., and Banks, J. (1787). "Observations on the structure and oeconomy of whales.," Philosophical Transactions of the Royal Society of London 77, 371-450.

Ing, R. K., Quieffin, N., Catheline, S., and Fink, M. (2005). "In solid localization of finger impacts using acoustic time-reversal process," Applied Physics Letters 87(20), 204104.

Keller, C. H., Hartung, K., and Takahashi, T. T. (1998). "Head-related transfer functions of the barn owl: measurement and neural responses," Hearing research 118(1), 13-34.

Ketten, D. (1994). "Functional analyses of whale ears: adaptations for underwater hearing," in OCEANS'94.'Oceans Engineering for Today's Technology and Tomorrow's Preservation.'Proceedings, IEEE, Vol. 1, pp. I-264.

Ketten, D. R. (1992). "The marine mammal ear: specializations for aquatic audition and echolocation," in The evolutionary biology of hearing (Springer), pp. 717-750.

Ketten, D. R. (1997). "Structure and function in whale ears," Bioacoustics 8(1-2), 103-135.

Ketten, D. R. (2000). "Cetacean ears," in Hearing by Whales and Dolphins (Springer New York, New York, NY), pp. 43-108. 
Kim, S., Kuperman, W., Hodgkiss, W., Song, H., Edelmann, G., and Akal, T. (2003). "Robust time reversal focusing in the ocean," The Journal of the Acoustical Society of America 114(1), 145-157.

Krysl, P., and Cranford, T. W. (2016). "Directional hearing and head-related transfer function in odontocete cetaceans," in The Effects of Noise on Aquatic Life II (Springer), pp. $583-587$.

Macpherson, E. A., and Sabin, A. T. (2013). "Vertical-plane sound localization with distorted spectral cues," Hearing research 306, 76-92.

McCormick, J. G., Wever, E., Palin, J., and Ridgway, S. (1970). "Sound conduction in the dolphin ear," The Journal of the Acoustical Society of America 48(6B), 1418-1428.

Møhl, B., Au, W., Pawloski, J., and Nachtigall, P. (1999). "Dolphin hearing: relative sensitivity as a function of point of application of a contact sound source in the jaw and head region," The Journal of the Acoustical Society of America 105(6), 3421-3424.

Mooney, T. A., Yamato, M., and Branstetter, B. K. (2012). "Hearing in cetaceans: from natural history to experimental biology," Adv. Mar. Biol 63(197-246).

Moore, P. W., and Au, W. W. (1975). "Underwater localization of pulsed pure tones by the california sea lion (z alophuscalifornianus)," The Journal of the Acoustical Society of America 58(3), 721-727.

Moore, P. W., Pawloski, D. A., and Dankiewicz, L. (1995). "Interaural time and intensity difference thresholds in the bottlenose dolphin (tursiops truncatus)," Sensory Systems of Aquatic Mammals 11-23. 
Mulsow, J., Finneran, J. J., and Houser, D. S. (2014). "Interaural differences in the bottlenose dolphin (tursiops truncatus) auditory nerve response to jawphone click stimuli," The Journal of the Acoustical Society of America 136(3), 1402-1409.

Nachtigall, P. E. (2016). "Biosonar and sound localization in dolphins," http: //neuroscience.oxfordre.com/view/10.1093/acrefore/9780190264086.001.0001/ acrefore-9780190264086-e-103, oxford Research Encyclopedia of Neuroscience.

Norris, K. S. (1964). "Some problems of echolocation in cetaceans," in Marine Bioacoustics, edited by W. N. Tavolga (Pergamon Press, New York), pp. 316-336.

Norris, K. S. (1968a). "The echolocation of marine mammals.," in The biology of marine mammals, edited by H. T. Andersen (Academic Press, New York), pp. 391-423.

Norris, K. S. (1968b). "The evolution of acoustic mechanisms in odontocete cetaceans," in Evolution and Environment, edited by E. T. Drake (Yale University Press, New York), pp. $297-324$.

Norris, K. S., and Harvey, G. W. (1974). "Sound transmission in the porpoise head," The Journal of the Acoustical Society of America 56(2), 659-664.

Popov, V. V., and Supin, A. Y. (1990). "Localization of the acoustic window at the dolphins head," in Sensory Abilities of Cetaceans (Springer), pp. 417-426.

Renaud, D. L., and Popper, A. N. (1975). "Sound localization by the bottlenose porpoise tursiops truncatus," Journal of Experimental Biology 63(3), 569-585.

Reysenbach de Haan, F. W. (1957). "Hearing in whales," Acta Oto-Laryngologica. Supplementum $134,1$. 
Richardson, W. J., Greene Jr, C. R., Malme, C. I., and Thomson, D. H. (2013). Marine mammals and noise (Academic press).

Ryabov, V. (2010). "Role of the mental foramens in dolphin hearing," Natural Science 2(6), 646-653.

Soldevilla, M. S., Henderson, E. E., Campbell, G. S., Wiggins, S. M., Hildebrand, J. A., and Roch, M. A. (2008). "Classification of rissos and pacific white-sided dolphins using spectral properties of echolocation clicks," The Journal of the Acoustical Society of America 124(1), 609-624.

Soldevilla, M. S., McKenna, M. F., Wiggins, S. M., Shadwick, R. E., Cranford, T. W., and Hildebrand, J. A. (2005). "Cuvier's beaked whale (ziphius cavirostris) head tissues: physical properties and ct imaging," Journal of experimental biology 208(12), 2319-2332. Song, Z., Zhang, Y., Thornton, S. W., Li, S., and Dong, J. (2017). "The influence of airfilled structures on wave propagation and beam formation of a pygmy sperm whale (kogia breviceps) in horizontal and vertical planes," The Journal of the Acoustical Society of America 142(4), 2443-2453.

Song, Z., Zhang, Y., Wei, C., and Wang, X. (2016). "Inducing rostrum interfacial waves by fluid-solid coupling in a chinese river dolphin (lipotes vexillifer)," Physical Review E 93(1), 012411.

Supin, A. Y., and Popov, V. V. (1993). "Direction-dependent spectral sensitivity and interaural spectral difference in a dolphin: Evoked potential study," The Journal of the Acoustical Society of America 93(6), 3490-3495. 
672

673 demic Press).

674

Van Opstal, J. (2016). The auditory system and human sound-localization behavior (Aca-

Wei, C., Au, W. W., Song, Z., and Zhang, Y. (2016). "The role of various structures in the head on the formation of the biosonar beam of the baiji (lipotes vexillifer)," The Journal of the Acoustical Society of America 139(2), 875-880.

Wei, C., Song, Z., Au, W. W., Zhang, Y., and Wang, D. (2018). "A numerical evidence of biosonar beam formation of a neonate yangtze finless porpoise (neophocaena asiaeorientalis)," Journal of Theoretical and Computational Acoustics 26(02), 1850009.

Youssef, K., Argentieri, S., and Zarader, J.-L. (2012). "Towards a systematic study of binaural cues," in Intelligent Robots and Systems (IROS), 2012 IEEE/RSJ International Conference on, IEEE, pp. 1004-1009. 